\title{
Identification géométrique des machines outils à structure parallèle : proposition d'une méthode basée sur l'usinage d'une pièce
}

\author{
Hélène Chanal ${ }^{1}$, Matthieu Rauch ${ }^{2}$, Emmanuel Duc ${ }^{1, a}$, Jean-Yves Hascoët ${ }^{2}$ \\ ET PASCAL RAY ${ }^{1}$ \\ 1 LaMI, IFMA/UBP, Campus de Clermont-Ferrand, les Cezeaux, BP 265, 63175 Aubière Cedex, France \\ 2 IRCCyN, UMR CNRS 6597, 1 rue de la Noë, BP 92101, 44321 Nantes Cedex, France
}

Reçu le 15 mars 2007, accepté le 6 juillet 2007

\begin{abstract}
Résumé - Un handicap important pour l'utilisation des machines outils à structure parallèle pour des opérations d'Usinage à Grande Vitesse $(\mathrm{UGV})$ se situe au niveau de la précision de pose de l'outil. Dans le cas des mécanismes à structure parallèle, ce défaut de pose est surtout dû aux erreurs liées à la transformation inverse de la commande. Ces erreurs peuvent être réduites à l'aide d'une identification des paramètres géométriques du modèle géométrique. Le but de cet article est de présenter différentes méthodes d'identification applicables aux machines outils à structure parallèle. Dans un premier temps, nous présentons les principes généraux de l'identification géométrique des machines outils à structure parallèle. Puis, nous décrivons une méthode statique issue de l'expérience acquise sur les machines outils sérielles utilisant un ball-bar 3D. Cette méthode est aujourd'hui appliquée sur la machine outil Verne qui se situe à l'IRCCyN (Nantes). Finalement, nous présentons une nouvelle méthode d'identification basée sur la mesure d'une pièce usinée. Cette méthode est réalisé en trois étapes : l'usinage d'une pièce dédiée, sa mesure sur une Machine à Mesurer Tridimensionnelle (MMT) et l'identification numérique des paramètres géométriques.
\end{abstract}

Mots clés : Machine à structure parallèle / identification géométrique / usinage à grande vitesse

\begin{abstract}
Geometrical calibration of parallel kinematics machines tools: Proposition of a new method based on part machining. A main limitation of Parallel Kinematics Machine tools (PKM) in high-speed machining tasks is their low level of tool pose accuracy. In case of parallel kinematics mechanisms, the pose defect is largely due to the inverse transformations realised by the controller. These errors can be reduced by identifying the geometrical parameters of the inverse kinematics model. The aim of this paper is to present different identification methods used for PKM. In a first time, the general principle of PKM geometrical identification is described. Then, a static method using a 3D ball-bar is presented from past experiences on serial machines tools. This method is applied today on the Verne machine tool located in IRCCyN (Nantes). Finally, a new calibration method based on the measure of a machined part is described. This method is implemented in three steps: machining of a dedicated part, measurement, and identification of the geometrical parameter values.
\end{abstract}

Key words: Parallel kinematics machine tool / geometrical calibration / high speed milling

\section{Introduction}

Actuellement, les machines outils à structure parallèle commencent à être utilisées dans l'industrie de l'automobile ou de l'aéronautique pour des opérations d'Usinage à Grande Vitesse (UGV). En effet, leurs performances dynamiques en terme de potentiel d'accélération

\footnotetext{
a Auteur pour correspondance : emmanuel.duc@ifma.fr
}

sont meilleures que celles des structures sérielles [1]. Cependant, un frein à leurs essors dans le domaine de l'usinage se situe au niveau du manque de précision de pose (position et orientation) de l'outil par rapport aux machines outils sérielles. Ce handicap est dû aux nombres importants de liaisons passives qui rendent délicate l'obtention d'un mécanisme précis [2].

La qualité de pose de l'outil peut être définie par deux grandeurs, l'exactitude de la pose et la répétabilité [3]. 


\section{Nomenclature}

\begin{tabular}{|ll|}
\hline$P_{i}\left(X_{\mathrm{m}} i, Y_{\mathrm{m}} i, Z_{\mathrm{m}} i\right)$ & point de contrôle de l'outil dans le repère $R_{\mathrm{m}}$ \\
$R_{0}\left(O_{\mathrm{p}}, x_{0}, y_{0}, z_{0}\right)$ & repère lié à l'espace de travail de la machine outil \\
$R_{\mathrm{m}}\left(O_{\mathrm{m}}, x_{\mathrm{m}}, y_{\mathrm{m}}, z_{\mathrm{m}}\right)$ & repère de mesure \\
$V=M G I(X, \xi)$ & modèle géométrique inverse \\
$V\left(V_{1}, V_{2}, V_{3}, B_{\alpha}, C_{\alpha}\right)$ & position des moteurs dans le repère articulaire \\
$V_{i}, V_{\mathrm{m}} i$ & pose théorique et mesurée des moteurs \\
$X(x, y, z, B, C)$ & position de l'outil dans le repère lié à l'espace de travail \\
$X_{\mathrm{d}}, X_{\mathrm{m}}$ & pose théorique et mesurée de l'outil \\
$\xi$ & paramètres géométriques \\
$\xi_{\mathrm{d}}$ & valeurs nominales des paramètres géométriques \\
$\phi$ & angle entre l'axe $\boldsymbol{x}_{0}$ de $R_{0}$ et $\boldsymbol{x}_{\mathrm{m}}$ de $R_{\mathrm{m}}$ \\
$(\Delta x, \Delta y, \Delta z)$ & coordonnées de $\overrightarrow{O_{\mathrm{p}} O_{\mathrm{m}}}$ dans $R_{0}$ \\
\hline
\end{tabular}

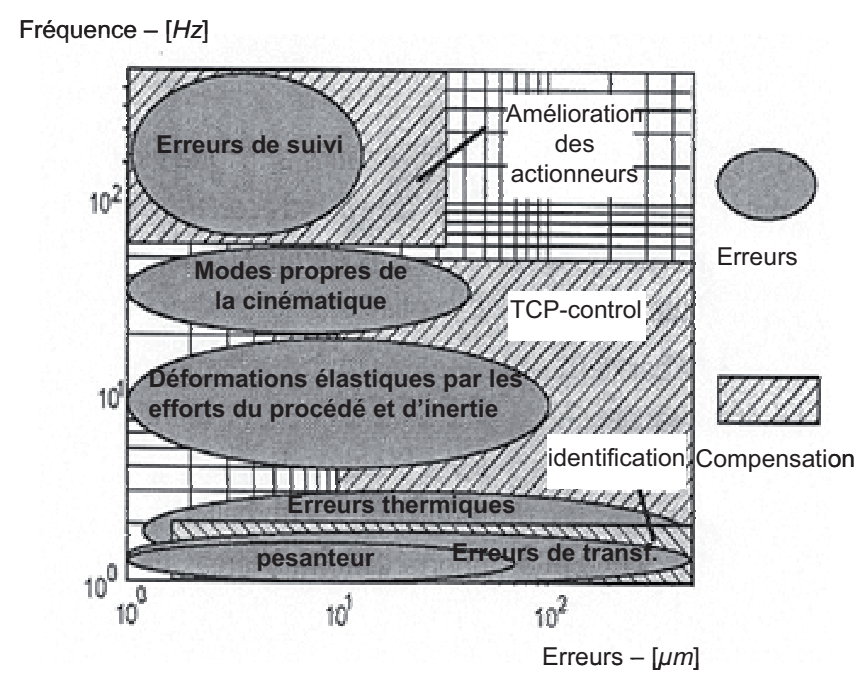

Fig. 1. Impacts du comportement de la machine sur les erreurs de position de l'outil [5].

D'après Merlet, la répétabilité des mécanismes à structure parallèle est élevée par rapport à l'exactitude de pose [4]. Dans la suite de cet article, nous négligeons le défaut de répétabilité par rapport au défaut d'exactitude de pose de l'outil. Nous appelons ce dernier défaut de précision.

Pour obtenir une amélioration de cette précision, plusieurs méthodes de compensation sont présentées dans la littérature. En effet, pendant l'usinage, le défaut de pose de l'outil est généré par différents comportements de la structure de la machine outil (Fig. 1). Une source d'erreur importante est la transformation inverse, nécessaire au pilotage des axes motorisés de la machine à partir des coordonnées du point piloté de l'outil. Ces erreurs peuvent être réduites à l'aide d'un étalonnage des paramètres géométriques du modèle géométrique inverse utilisé pour la commande $[5,6]$. Les méthodes les plus précises sont celles utilisant des mesures externes, dite extéroceptives. Ainsi, la pose réelle de l'outil est mesurée et comparée avec la pose théorique, afin de déterminer les paramètres géométriques permettant de réduire l'erreur entre les deux estimations.

Cependant, dans le cadre de l'usinage, la qualité d'une pièce usinée ne dépend pas directement du défaut de pose de l'outil [7]. Ainsi, nous estimons qu'une identification est correcte si :

- l'identification permet à la pièce d'être usinée avec la qualité requise ;

- le temps pris par l'identification est court (de l'ordre de quelques heures) ;

- la méthode d'identification utilise des outils de mesure simples et connus.

Le but de cet article est de présenter dans un premier temps une méthode issue de l'expérience acquise sur les machines sérielles. Ainsi, grâce à différentes mesures avec un ball-bar 3D, il est possible de mettre en relation la position des axes machines et la position de l'effecteur dans l'espace de travail. Ainsi, le modèle géométrique est identifié.

Dans un second temps, nous présentons une méthode basée sur la mesure d'une pièce usinée. Cette méthode est réalisée en trois étapes : l'usinage d'une pièce dédiée, sa mesure sur une Machine à Mesurer Tridimensionnelle (MMT) et l'identification numérique des valeurs des paramètres géométriques. Ainsi, l'identification est réalisée en prenant en compte la trajectoire suivie par l'outil et non pas les différentes poses prises par l'outil. En effet, en usinage, ce n'est pas la pose de l'outil qui fait la surface mais l'enveloppe des différentes poses prises par l'outil tout au long d'une trajectoire.

Nous illustrons les propos de cet article sur la machine outil Verne qui se situe à l'IRCCyN (Nantes). Nous commençons donc par la présenter. Puis, nous détaillons les principes généraux de l'identification géométrique des machines outils à structure parallèle en décrivant plus particulièrement les méthodes usuelles de mesure extéroceptive. Nous décrivons plus particulièrement une méthode de mesure utilisant un ball-bar 3D. Finalement, nous présentons notre méthode d'identification en décrivant la forme de la pièce usinée, son usinage et sa mesure, pour terminer, sur la méthode numérique d'identification.

\section{Présentation de la machine outil Verne}

Développée par la société espagnole Fatronik, la machine outil Verne est un centre d'usinage 5 axes à 


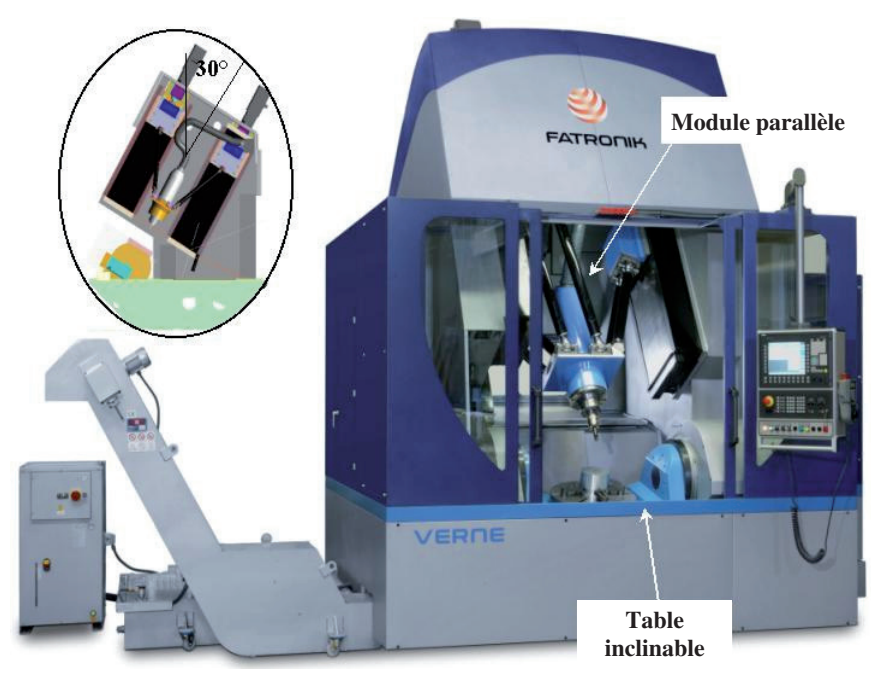

Fig. 2. Vue générale de la machine outil Verne.

structure parallèle. Sa principale caractéristique est que sa structure combine un module parallèle, qui exécute les translations selon les axes $x, y$ et $z$ et un module sériel, qui fournit les rotations autours des axes $B$ et $C$. Il s'agit donc d'une machine hybride (Fig. 2). L'axe de la broche est incliné de $30^{\circ}$ par rapport à la verticale et le volume de travail est exempt de toute singularité. Les capacités d'usinage de la Verne sont les suivantes : vitesse de rotation $24000 \mathrm{tr} . \mathrm{min}^{-1}$, puissance de broche $40 \mathrm{~kW}$, avance maximale $50 \mathrm{~m} \cdot \mathrm{min}^{-1}$ et accélération maximale $15 \mathrm{~m} \cdot \mathrm{s}^{-2}$.

Une représentation schématique du module parallèle est donnée sur la figure 3. Celui-ci est constitué de six jambes de longueur constante $\left(A_{i} B_{i}\right)$, reliées deux à deux à trois moteurs $\left(V_{1}, V_{2}, V_{3}\right)$. En commandant la position de chacun des trois moteurs, le Directeur de Commande Numérique (DCN) peut ainsi contrôler la position du centre outil dans l'espace de travail.

Une autre spécificité de la Verne est qu'un modèle de transformation géométrique a été implémenté et intégré à la commande numérique pour traduire les instructions de déplacement du programme d'usinage (en coordonnées cartésiennes) en déplacement des axes réels (en coordonnées articulaires). Ceci permet aussi de compenser les défauts de rectitude et de perpendicularité. Ce modèle est fonction de paramètres géométriques à identifier pour diminuer l'erreur de transformation inverse.

\section{Principes d'identification des machines outils à structure parallèle}

L'étalonnage ou l'identification d'une machine outil à structure parallèle consiste en la détermination d'un modèle qui décrit au mieux le mécanisme. Trois étapes principales doivent être contrôlées pendant l'identification :

- la modélisation géométrique du mécanisme;

- la méthode d'identification des paramètres géométriques, ainsi que la fonction erreur associée;
- le choix de la méthode expérimentale de mesure, ainsi que ses outils.

\subsection{Modélisation géométrique du mécanisme}

Le choix du modèle géométrique utilisé lors de l'identification d'un mécanisme se fait en analysant l'influence des paramètres géométriques sur la pose de l'effecteur. Une analyse de sensibilité est souvent utilisée pour déterminer les paramètres géométriques les plus influents d'après Fan [8]. Cette analyse permet aussi de justifier le choix des paramètres géométriques du modèle géométrique inverse utilisé dans la commande et de vérifier leur indépendance et identifiabilité $[9,10]$.

Cette analyse permet de valider le choix des 42 paramètres géométriques servant à la modélisation de la structure parallèle de la Verne implémentée dans sa commande par le constructeur. Notons que ce modèle géométrique semble avoir été réalisé suivant la méthode proposée par Merlet [4]. D'autres paramètres géométriques ont été ajoutés par le constructeur afin de prendre en compte le module sériel et la position du module sériel par rapport au parallèle. Toutefois, ces derniers sont identifiés avec une méthode différente de celle appliquée au module parallèle. Dans la suite, nous nous focalisons uniquement sur le module parallèle.

\subsection{Choix de la fonction erreur}

La modélisation géométrique du mécanisme définit les paramètres géométriques à identifier. Ils sont identifiés à partir d'informations qui permettent de confronter deux estimations de l'état du mécanisme [11]. Ces informations sont contradictoires si les paramètres géométriques ne décrivent pas correctement le mécanisme. Dans la plupart des cas, les paramètres géométriques sont identifiés en minimisant une fonction erreur comparant ces deux estimations. Cette fonction erreur dépend du moyen utilisé pour obtenir la redondance d'informations. En effet, certains capteurs mesurent la position des liaisons du mécanisme dans l'espace articulaire, d'autres mesurent la pose de l'effecteur dans l'espace de travail.

Dans le cas d'un étalonnage externe comme nous souhaitons le réaliser, Renaud compare l'état du système dans l'espace articulaire afin d'utiliser le modèle géométrique inverse et d'obtenir une fonction erreur analytique [11]. Généralement, cette fonction erreur est de type « moindres carrés ».

Dans le cas de la Verne, le modèle géométrique inverse exprime la position des moteurs $V$ dans le repère articulaire en fonction de la position et de l'orientation de l'outil $X$ dans le repère cartésien lié à l'espace de travail et des paramètres géométriques identifiés $\xi$ :

$$
V=M G I(X, \xi) \text {. }
$$

Ainsi, pour comparer la pose de la machine dans le repère articulaire, les positions théoriques des moteurs $V_{i}$ sont 

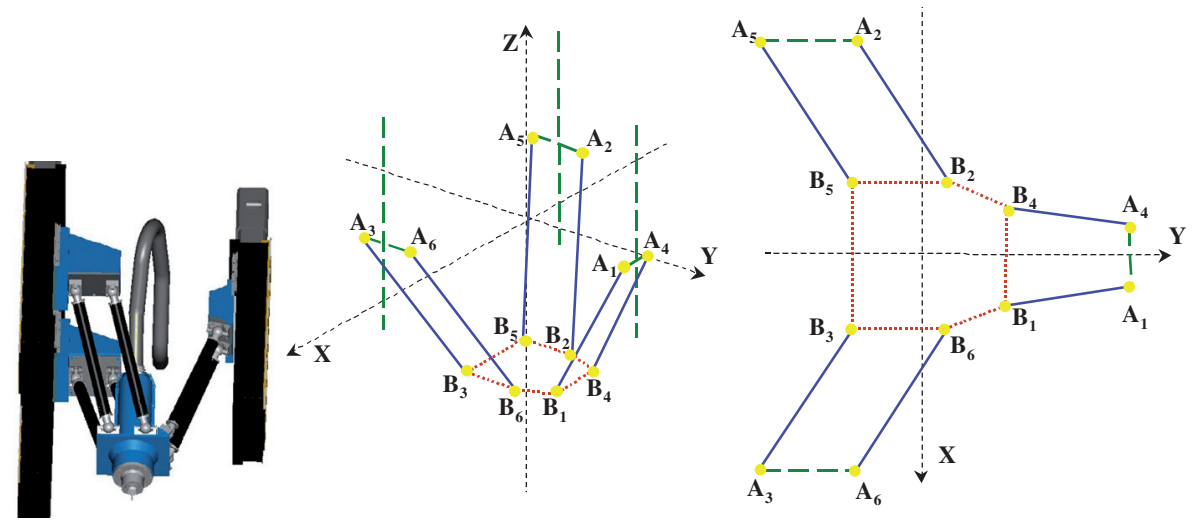

Fig. 3. Module parallèle de la Verne.

comparées à celles estimées $V_{\mathrm{m} i}$, obtenues à partir des poses mesurées $X_{\mathrm{m} i}$ de l'outil (Fig. 4). Les positions théoriques $V_{i}$ associées des moteurs sont calculées pour des poses théoriques $X_{\mathrm{d} i}$ de l'outil et des valeurs nominales des paramètres géométriques $\xi_{\mathrm{d}}$. L'identification des paramètres géométriques $\xi$ est réalisée si $\boldsymbol{V}_{i}$ et $\boldsymbol{V}_{\mathrm{m} i}$ sont égaux dans le cas de comparaisons ponctuelles :

$$
\begin{aligned}
f_{\text {erreur }}(\xi) & =\min _{\xi}\left(\sum_{i=1}^{n}\left\|V_{i}-V_{\mathrm{m} i}\right\|^{2}\right) \\
& =\min _{\xi}\left(\sum_{i=1}^{n}\left\|M G I\left(X_{\mathrm{d} i}, \xi_{\mathrm{d}}\right)-M G I\left(X_{\mathrm{m} i}, \xi\right)\right\|^{2}\right)
\end{aligned}
$$

où $n$ est le nombre de poses mesurées.

Les paramètres géométriques identifiés $\xi$ sont ceux qui minimisent cette fonction erreur.

\subsection{Méthode de mesure de pose usuelle pour les machines outils à structure parallèle}

Il existe plusieurs types de méthodes expérimentales pour identifier une machine outil à structure parallèle. Certaines sont réalisées ponctuellement à l'aide d'appareil externe ou d'une pièce mesurée $[5,9,12,13]$. D'autres sont conduites à l'aide d'appareils de mesure interne montés sur la structure de la machine [5]. Le second type de méthode permet de corriger la machine pendant l'usinage et de compenser les erreurs thermiques, de pesanteur et dynamiques. Dans la suite de ce paragraphe, nous nous intéressons plus particulièrement à la réalisation de mesures extéroceptives soit en utilisant un appareillage dédié via l'usinage d'une pièce.

\subsubsection{Méthode utilisant un appareillage dédié}

Une première méthode pour identifier une machine à structure parallèle consiste en l'ajout d'une contrainte mécanique limitant les déplacements de l'outil afin de mesurer des défauts de pose de l'outil. Ainsi, Weck réalise une redondance de mesure de la position de l'outil à l'aide d'un axe linéaire attaché au bâti par un joint de cardan et relié à la broche par un cône HSK [9]. Ce système ressemble aux solutions de Martinez utilisant un mécanisme ball-bar [13].

Ce mécanisme ball-bar 3D est celui utilisé sur la Verne. Une première étape de la calibration vise à assurer une bonne perpendicularité des axes de la machine et un bon centrage de l'axe de la broche, par rapport au plateau de la machine. Ensuite, la méthode utilisée par Fatronik nécessite l'emploi d'un ball-bar pour affiner les réglages obtenus précédemment d'une part et d'autre part pour assurer un positionnement acceptable de l'outil quelle que soit la configuration de la zone de travail.

En pratique, l'une des extrémités du ball-bar est fixée à un point de la table dont la position dans l'espace de travail est connue; l'autre est fixée à un porte-outil muni d'une pince montée en broche, simulant ainsi le centre d'un outil (Fig. 5). Un programme CN commande ensuite le déplacement des axes de la machine afin que le centre outil parcoure 324 points, tous situés sur une demisphère dont le rayon est égal à la longueur théorique du ball-bar et dont le centre est le point de la table évoqué précédemment. À chacun de ces 324 points, la machine s'arrête et la longueur réelle du ball-bar est acquise. On obtient donc un fichier contenant les 324 longueurs de ball-bar pour les 324 points programmés. Cette opération est répétée pour 3 points de la table et pour deux longueurs d'outils différentes. On obtient finalement 6 fichiers d'acquisition.

Les résultats des mesures, ainsi que les paramètres géométriques de transformations actuels, sont injectés dans une routine développée par Fatronik spécialement pour la Verne. Cette routine génère alors un exécutable qui, une fois dans la $\mathrm{CN}$, met à jour les paramètres géométriques (Fig. 6). Si la précision obtenue n'est pas satisfaisante, il faudra répéter l'étape d'étalonnage au ball-bar. Dans la pratique, deux ou trois itérations de calibration ball-bar sont nécessaires pour une précision acceptable.

D'autres méthodes consistent en l'utilisation d'un capteur laser extéroceptif. Ainsi, Song utilise un laser 5D qui peut mesurer suivant 3 axes de déplacement linéaire et 2 axes de déplacement angulaire [12]. 


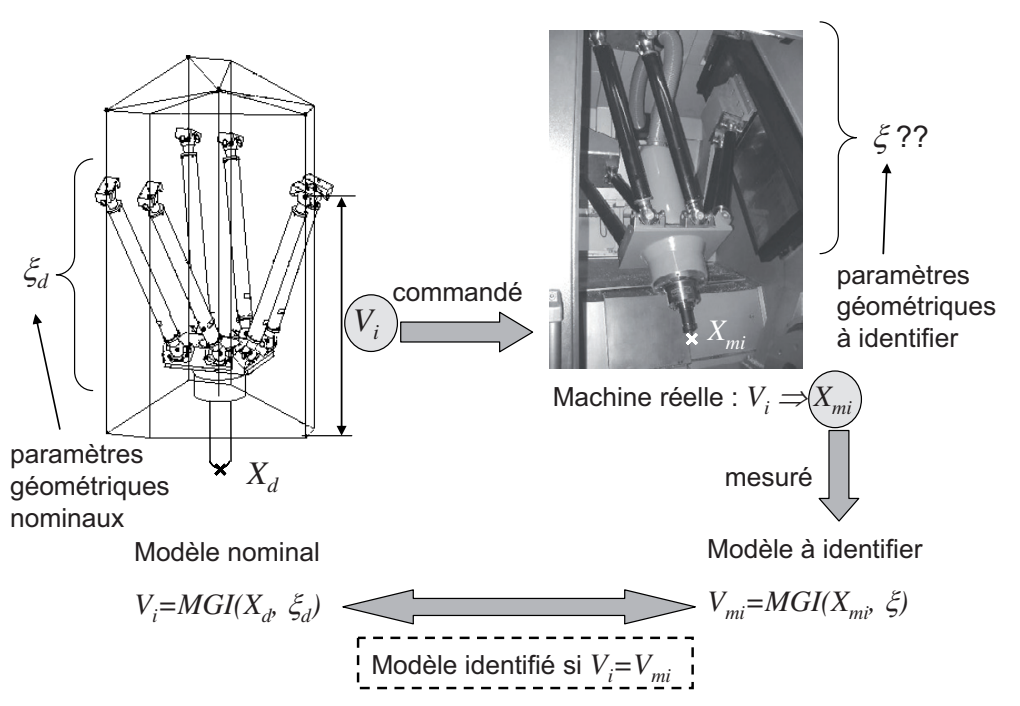

Fig. 4. Comparaison dans l'espace articulaire de la pose de la machine outil.

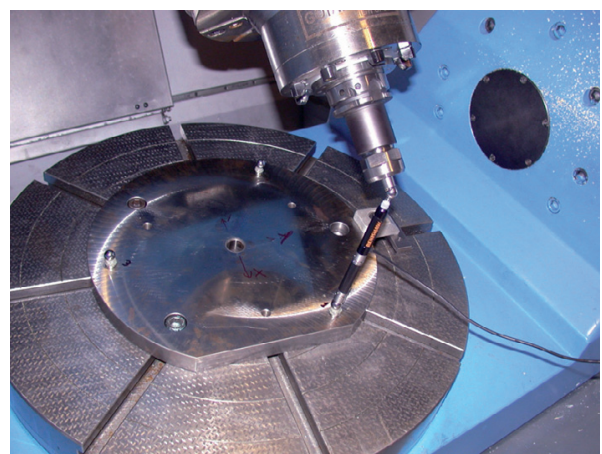

Fig. 5. Acquisition ball-bar lors d'une calibration de la Verne.

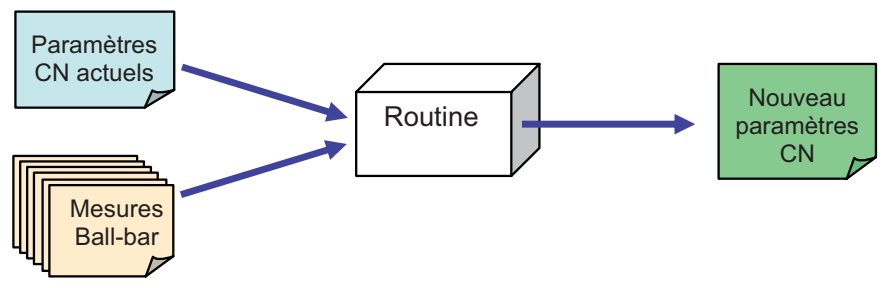

Fig. 6. Principe de fonctionnement de la calibration « Fatronik».

Cependant, les méthodes présentées précédemment sont des moyens de mesure qui sont directement issus de la robotique ou de l'identification des machines outils sérielles. Elles nécessitent l'immobilisation de la machine, les outils de mesure utilisés sont onéreux et dédiés à l'identification et, une certaine expertise de l'utilisateur afin de réaliser une identification correcte. Ces méthodes peuvent donc être qualifiées de «méthodes de laboratoire qui ne sont pas adaptées à la fonction principale de la machine outil, c'est-à-dire produire des pièces ayant une qualité suffisante avec des contraintes du monde industriel. Il est donc pertinent de développer des méthodes adaptées plus particulièrement à l'usinage avec des machines outils

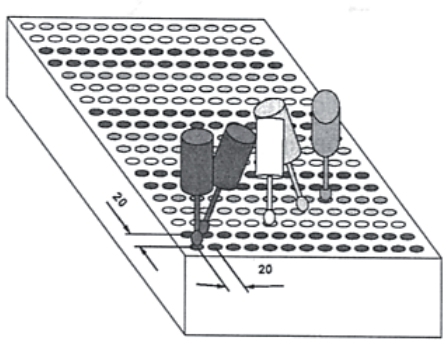

Fig. 7. Pièce usinée par Pritschow [5].

à structure parallèle en utilisant des moyens usuels de métrologie.

\subsubsection{Méthode utilisant l'usinage d'une pièce}

Pour prendre en compte cette contrainte, la mesure d'une pièce usinée devient intéressante. Ainsi, Pritschow usine des calottes sphériques avec un outil hémisphérique suivant plusieurs positions et orientations de l'outil afin de solliciter tous les paramètres géométriques [5] (Fig. 7).

Après l'usinage de sa pièce, Pritschow détermine le centre de la sphère inscrite dans chaque calotte, et il considère que le centre de l'outil hémisphérique était en ce point au moment de l'usinage. Après la détermination de l'ensemble des positions prises par l'outil, il les compare à celles désirées afin de réaliser l'identification des paramètres géométriques de la machine.

La conception de la forme de cette pièce peut donc être améliorée. Les calottes usinées permettent de déterminer la position réelle de l'outil et de la comparer avec la position théorique comme avec les méthodes décrites précédemment. Toutefois, plusieurs surfaces continues sont usinées pendant une phase d'usinage. Ainsi, il devient intéressant de développer une méthode permettant de comparer la trajectoire réelle de l'outil ayant généré 


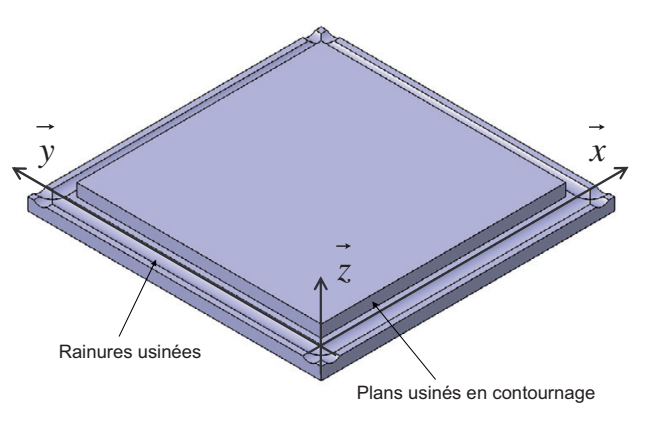

Forme de la pièce usinée

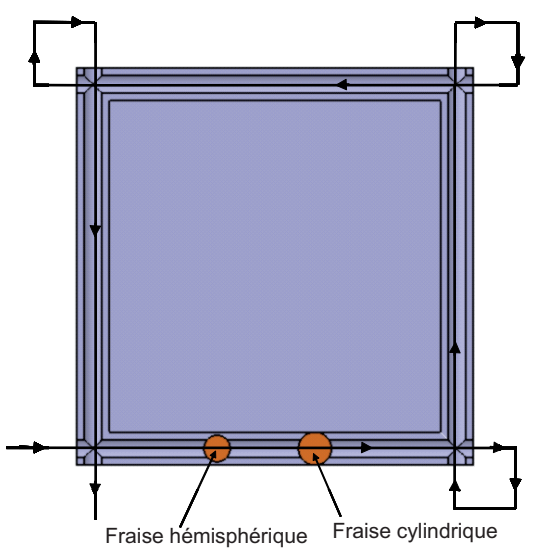

trajectoire

Fig. 8. Pièce dédiée à l'identification.

la surface usinée avec la trajectoire théorique qu'il aurait due suivre. Ainsi, la méthode d'identification est plus en adéquation avec l'utilisation des machines outils.

De plus, même si les méthodes actuelles permettent de minimiser le défaut de pose de l'outil et donc d'usiner des pièces avec la qualité requise, elles sont difficiles et longues à mettre en ouvre de manière rigoureuse. Dans un contexte de fabrication, une méthode permettant d'identifier les paramètres géométriques dans une partie de l'espace de travail pour une forme de pièce à usiner doit permettre d'augmenter le gain en précision apporté par l'identification et de diminuer le temps d'immobilisation de la machine outil.

\section{Méthode d'identification des paramètres géométriques avec l'usinage d'une pièce}

Dans la section précédente, nous avons donc détaillé les différentes étapes nécessaires à la réalisation de l'identification. Dans la suite, nous allons nous attacher à définir une nouvelle méthode d'identification externe du module parallèle par l'usinage d'une pièce.

\subsection{Conception de la forme de la pièce usinée}

La méthode d'identification choisie consiste en l'usinage d'une pièce suivie de sa métrologie afin d'aboutir à l'identification des paramètres géométriques du modèle de la commande. Cependant, la méthode expérimentale doit répondre aux contraintes d'un étalonnage géométrique, c'est-à-dire que les seuls phénomènes générés par les erreurs de transformations inverses doivent apparaître sur la pièce usinée. De plus, tous les paramètres géométriques doivent avoir une influence sur la qualité de la pièce usinée.

Ainsi, une pièce en alliage d'aluminium (7075) réalisée à l'aide d'opérations de contournage avec une fraise deux tailles de diamètre $20 \mathrm{~mm}$ et d'opérations de rainurage avec une fraise hémisphérique de diamètre $16 \mathrm{~mm}$ permet de répondre à ces contraintes [14] (Fig. 8). Les trajectoires pour usiner ces surfaces sont des lignes droites. En effet, dans le cas des machines outils à structure parallèle ayant un comportement anisotrope, tous les paramètres géométriques ont une influence lorsque l'outil se déplace suivant une ligne droite. Aussi, tous les paramètres géométriques peuvent être correctement identifiés.

Les dimensions et la position de la pièce dédiée à l'identification doivent être adaptées aux pièces qui sont usinées sur la machine étalonnée. En effet, si la pièce dédiée est trop petite ou mal positionnée dans l'espace de travail, nous ne pouvons garantir le respect de la qualité requise. De même réaliser une pièce trop grande diminue la précision apportée par l'identification. Afin de pouvoir mesurer la pièce, nous estimons que le contournage doit être réalisé sur $10 \mathrm{~mm}$ au moins et que la profondeur de la rainure doit être au moins de $2 \mathrm{~mm}$.

Sur la figure 8, nous pouvons remarquer que les trajectoires pour l'usinage de la rainure et du contournage sont identiques afin de pouvoir utiliser les deux formes pour déterminer la position du point piloté de l'outil à un instant donné. Ainsi, la mesure du plan contourné nous permet de déterminer le défaut de position de l'outil dans un plan vertical perpendiculaire à ce dernier. La mesure de la surface rainurée nous amène à connaître la position verticale du point piloté de l'outil.

\subsection{Détermination de la pose de l'outil}

Pour réaliser l'identification, la pose de l'outil doit être déterminée dans un repère de dégauchissage $R_{\mathrm{m}}$ en fonction des mesures des surfaces usinées (Fig. 9). Ce repère de dégauchissage est défini de telle manière que les surfaces ayant servi à la mise en position de la pièce sur la table de la machine outil servent à sa détermination.

Considérons un plan qui coupe perpendiculairement au trajet de l'outil le plan usiné en contournage et la rainure. Dans ce plan, notons $P_{i}\left(X_{\mathrm{m} i}, Y_{\mathrm{m} i}, Z_{\mathrm{m} i}\right)$ le point piloté de l'outil dans le repère de dégauchissage $R_{\mathrm{m}}$. La 


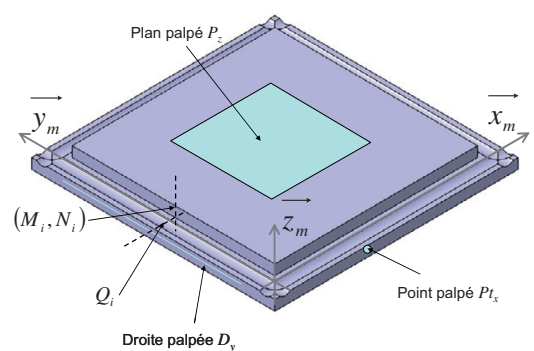

Repère de dégauchissage

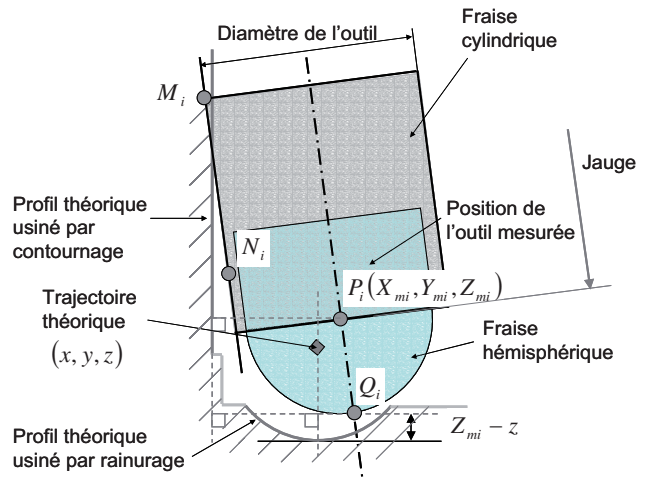

Calcul défaut de position

Fig. 9. Identification de la pose de l'outil.

coordonnée $Z_{\mathrm{m} i}$ du centre de l'outil est déterminée en mesurant la position du point $Q_{i}$, au fond de la rainure. Deux points $M_{i}$ et $N_{i}$ sont palpés sur le plan obtenu en contournage. Si nous considérons que ces points $M_{i}$ et $N_{i}$ sont usinés au même instant, nous pouvons déterminer la coordonnée $X_{\mathrm{m} i}$ du point piloté de l'outil si le plan théorique est usiné suivant l'axe $y$ ou $Y_{\mathrm{m} i}$ si c'est suivant l'axe $x$ (Fig. 9).

Ainsi, les mesures réalisées sur la MMT nous permettent de déterminer plusieurs positions de l'outil dans le repère de dégauchissage $R_{\mathrm{m}}$. Pour réaliser l'identification, les positions de l'outil mesurées doivent être exprimées dans ce repère cartésien $R_{0}$, lié à l'espace de travail de la machine outil. Nous faisons l'hypothèse que l'axe $z$ de $R_{0}$ est identique à l'axe $z_{\mathrm{m}}$ de $R_{\mathrm{m}}$. Ainsi, un point $P_{i}$ connu dans le repère $R_{\mathrm{m}}$ peut s'exprimer dans le repère $R_{0}$ en fonction du vecteur $\overrightarrow{\mathbf{O}_{\mathbf{p}} \mathbf{O}_{\mathbf{m}}}(\Delta x, \Delta y, \Delta z)$ $\left(O_{\mathrm{p}}\right.$ est l'origine du repère $R_{0}$ et $O_{\mathrm{m}}$ l'origine de $\left.R_{\mathrm{m}}\right)$ et de l'angle $\phi=\left(\rightarrow x, \rightarrow x_{\mathrm{m}}\right)$.

Nous devons donc, maintenant, exprimer la fonction erreur permettant de comparer la trajectoire théorique suivie par l'outil à la trajectoire réelle ayant généré les surfaces mesurées. Les paramètres optimisés dans cette fonction erreur sont donc les 42 paramètres géométriques $\xi$, les coordonnées du vecteur $\vec{O}_{\mathrm{p}} O_{\mathrm{m}}(\Delta x, \Delta y, \Delta z)$ et l'angle $\phi$.

\subsection{Mise en œuvre numérique de l'identification}

Pour chaque position $P_{i}$ de l'outil, à l'aide du modèle géométrique inverse à identifier, nous pouvons exprimer la position des moteurs de la machine dans le repère articulaire en faisant l'hypothèse que l'orientation de l'outil est parfaite :

$$
V_{\mathrm{m} i}=M G I\left(P_{\mathrm{i}}, \xi\right) .
$$

Comme, nous identifions les paramètres géométriques décrivant la structure parallèle, nous nous intéressons plus particulièrement aux positions $V_{\mathrm{m} 1 i}, V_{\mathrm{m} 2 i}$ et $V_{\mathrm{m} 3 i}$ des moteurs verticaux. En effet, nous considérons la partie sérielle correctement identifiée par un étalonnage dédié. Ainsi, nous obtenons un ensemble de points définissant différentes poses de la structure parallèle de la machine qui doivent être confondues avec la trajectoire théorique commandée dans l'espace articulaire pour que l'identification soit réalisée.

Afin de réaliser l'identification, nous définissons la

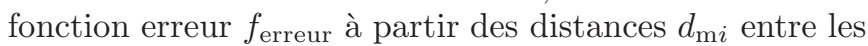
points définis avec les coordonnées $\left(V_{\mathrm{m} 1 i}, V_{\mathrm{m} 2 i}, V_{\mathrm{m} 3 i}\right)$ et la trajectoire commandée dans l'espace articulaire :

$$
f_{\text {erreur }}=\min _{\xi, \Delta x, \Delta y, \Delta z, \phi} \sum_{i=1}^{n} d_{\mathrm{m} i}(\xi, \Delta x, \Delta y, \Delta z, \phi)^{2}
$$

Nous considérons que les paramètres $\xi, \Delta x, \Delta y, \Delta z$ et $\phi$ sont identifiés lorsqu'ils minimisent $f_{\text {erreur. Ainsi, la tra- }}$ jectoire commandée dans l'espace articulaire correspond à la trajectoire souhaitée dans l'espace de travail.

\section{Conclusion}

Le but de cet article était de présenter des méthodes d'identification extéroceptive de machines outils à structure parallèle. Ainsi, le système ball-bar 3D utilisé sur la machine-outil Verne est détaillé. Cependant cette méthode de mesure de position de l'outil que nous pouvons qualifier de « laboratoire » n'est pas adaptée dans un contexte industriel. Nous avons donc proposé une autre méthode qui consiste en l'usinage d'une pièce, sa mesure sur une MMT puis l'identification numérique des paramètres géométriques. Cette nouvelle méthode prend en compte une fonction principale des machines-outils qui est de produire des pièces ayant la qualité requise.

Cette nouvelle méthode a été appliquée à la Verne. Elle nécessite 6 heures de mise en œuvre, 2 heures pour réaliser l'usinage et la métrologie et 4 heures de calculs numériques. À ce jour, elle permet d'améliorer la précision d'usinage par rapport à la pièce test. Ainsi les dimensions produites par des usinages suivant l'axe $x$ sont respectées avec une précision $<0,02 \mathrm{~mm}$ et suivant $y<0,04 \mathrm{~mm}$. Il est maintenant intéressant de comparer cette méthode avec un système ball-bar afin de quantifier le gain qu'elle apporte. 
Remerciements. Ces travaux de recherche s'inscrivent dans le cadre de la Fédération de Recherches TIMS/CNRS 2856 financée par le Conseil Régional d'Auvergne, le Ministère de la recherche, le CNRS et le CEMAGREF.

Ce travail a été conduit dans le cadre du groupe de travail Manufacturing 21 qui regroupe 11 laboratoires de recherche français. Les thèmes étudiés sont :

- la modélisation du processus de fabrication,

- l'usinage virtuel,

- le développement de nouvelles méthodes de fabrication.

\section{Références}

[1] M. Terrier, A. Dugas, J.Y. Hascoët, Qualification of parallel kinematics machine in high speed milling on free form, Int. J. Machine Tool Manufacture 44 (2004) 865877

[2] W. Khalil, E. Dombre, Modélisation, identification et commande des robots, Hermès, $\mathrm{N}^{\circ}$ ISBN 2-7462-0003-1, 1999

[3] M. Priel, Les robots industriels : caractéristique, performance et choix, Collection AFNOR Technique, $\mathrm{N}^{\circ}$ ISBN 2-1230-6211-3, 1990

[4] J.P. Merlet, Les robots parallèles, Hermès, $N^{\circ}$ ISBN 286601-599-1, 1997

[5] G. Pritschow, C. Eppler, T. Garber, Influence of the dynamic stiffness on the accuracy of PKM, 3rd Chemnitz Parallel Kinematic Seminar, Chemnitz, Allemagne, mai 2002, pp. 313-333

[6] T. Huang, D.G. Chetwynf, D.J. Whitehouse, J. Wang, A general and novel approach for parameter identification of a 6-DOF parallel kinematic machines, Mechanism and Machine Theory 40 (2005) 219-239
[7] H. Chanal, E. Duc, P. Ray, A study of the impact of machine tool structure on machining processes, Int. J. Machine Tool Manufacture 46 (2006) 98-106

[8] K.C. Fan, H. Wang, J.W. Zhao, T.H. Chang, Sensitivity analysis of the 3-PRS parallel kinematic spindle platform of a serial-parallel machine tool, Int. J. Machine Tool Manufacture 43 (2003) 1561-1569

[9] M. Weck, D. Staimer, On the accuracy of parallel kinematic machine tools: design, compensation and calibration, 2rd Chemnitz Parallel Kinematic Seminar, Chemnitz, Allemagne, janvier 2000, pp. 73-84

[10] H. Chanak, E. Duc, P. Ray, Calibration accuracy of a parallel structure machine tool with respect to machine part quality, 6th International Conference on Integrated Design and Manufacturing in Mechanical Engineering, Grenoble, France, mai 2006

[11] P. Renaud, Apport de la vision pour l'identification géométrique de mécanisme parallèle, thèse, Université Blaise Pascal, Clermont-Ferrand, 2003

[12] J. Song, J.I. Mou, C. King, Error modelling and compensation for parallel kinematic machines, in C.R. Boer, L. Molinari-Tosatti, K.S. Smith, Parallel kinematic machines : theoretical aspects and industrial requirement, Springer-Verlag edition, $N^{\circ}$ ISBN 1-85233-613-7, 1999, pp. $170-187$

[13] L. Martinez, V. Collado, Calibration of a hybrid serial/parallel 5-axes milling machine using a double bar probe, 3rd Chemnitz Parallel Kinematic Seminar, Chemnitz, Allemagne, mai 2002, pp. 137-150

[14] H. Chanal, E. Duc, P. Ray, J.Y. Hascoët, Design of a machined dedicated part for calibrating parallel kinematics machine tool, $5^{\mathrm{e}}$ Congrés international Usinage grande vitesse, Metz, France, mars 2006 